\title{
Oropharyngeal flora and chest infection after upper abdominal surgery
}

\author{
J P Dilworth, R J White, E M Brown
}

\begin{abstract}
The oropharyngeal flora was determined before and after operation in 127 patients undergoing upper abdominal surgery. Swabs of the oropharynx were obtained on the day before operation and on the first, third, and fifth postoperative days. Isolation of Haemophilus infuenzae, Streptococcus pneumoniae, and coliforms was noted. In the 108 patients with the full series of throat swabs the incidence of oropharyngeal colonisation by $H$ influenzae was $16 \%$ and was unchanged after operation. $S$ pneumoniae was present in only 5.6 (six patients) before operation and the incidence fell to $1.9 \%$ (two patients). There was a transient rise in coliform colonisation postoperatively. Twenty four patients developed a chest infection. In eight a bacterial cause was established, in six $H$ influenzae and in two $S$ pneumoniae. There was a significant relation between the carriage of $H$ influenzae before operation and development of a chest infection. $H$ influenzae was also found more often in cigarette smokers. The presence of $\boldsymbol{S}$ pneumoniae or coliform organisms before surgery was not related to the development of infection. The high incidence of postoperative chest infection in cigarette smokers appears to be due in part to preoperative colonisation of the oropharynx by $H$ influenzae.
\end{abstract}

The incidence of chest infection after upper abdominal surgery under general anaesthesia varies from $17 \%$ to $38 \%$. $^{1-4}$ The bacteria most commonly concerned, particularly Haemophilus influenzae, may be part of the normal flora of the oropharynx. ${ }^{56}$ If these organisms

Accepted 6 December 1990 are aspirated during or after the operative procedure they will encounter the conditions of reduced clearance ${ }^{7}$ and impaired host defences that allow colonisation. The presence of these potential pathogens in the oropharynx before operation might therefore be related to the development of postoperative chest infection. We tested this hypothesis by determining the oropharyngeal flora before and after operation in patients undergoing upper abdominal surgery and its relation to chest infection.

\section{Methods}

We studied 127 consecutive patients undergoing elective upper abdominal surgery during 12 months.

Throat swabs were obtained by sampling the pharyngeal mucosa bilaterally with the tongue depressed. Specimens were collected on the day before surgery and on the first, third, and fifth postoperative days. Over $90 \%$ of the swabs were taken by one individual (JPD) and the remainder by nursing staff using the same procedure. All swabs were inoculated directly on to the following media within 45 minutes of collection: blood agar, chocolate agar on which a bacitracin disc was placed or in which bacitracin $(10 \mathrm{IU} / \mathrm{ml})$ was incorporated, ${ }^{8}$ and CLED (cysteine lactose electrolyte deficient) agar. All plates were incubated overnight at $37^{\circ} \mathrm{C}$ in a carbon dioxide incubator. Isolates were identified according to standard laboratory procedures. Pure or heavy growths of $H$ influenzae and Streptococcus pneumoniae were noted.

Each patient was assessed clinically on the day immediately preceding surgery, on three of the four postoperative days, and on alternate days thereafter until discharge. The presence of cough, the degree of purulence of sputum, body temperature, and physical signs in the chest were recorded. A chest radiograph and the blood white cell count were also obtained when any of these symptoms or signs were present. Chest infection was diagnosed by the presence of four of the following features: fever, cough, purulent sputum, chest signs, leucocytosis, and radiographic changeexcept when there was purulent sputum, when only one other criterion was required (table 1).

Patients were defined as smokers (20 or more pack years) or non-smokers (less than 20 pack years). Preoperative spirometry was performed; airflow obstruction was defined as an FEV $_{1}$ :FVC ratio below $70 \%$. Chronic bron- 


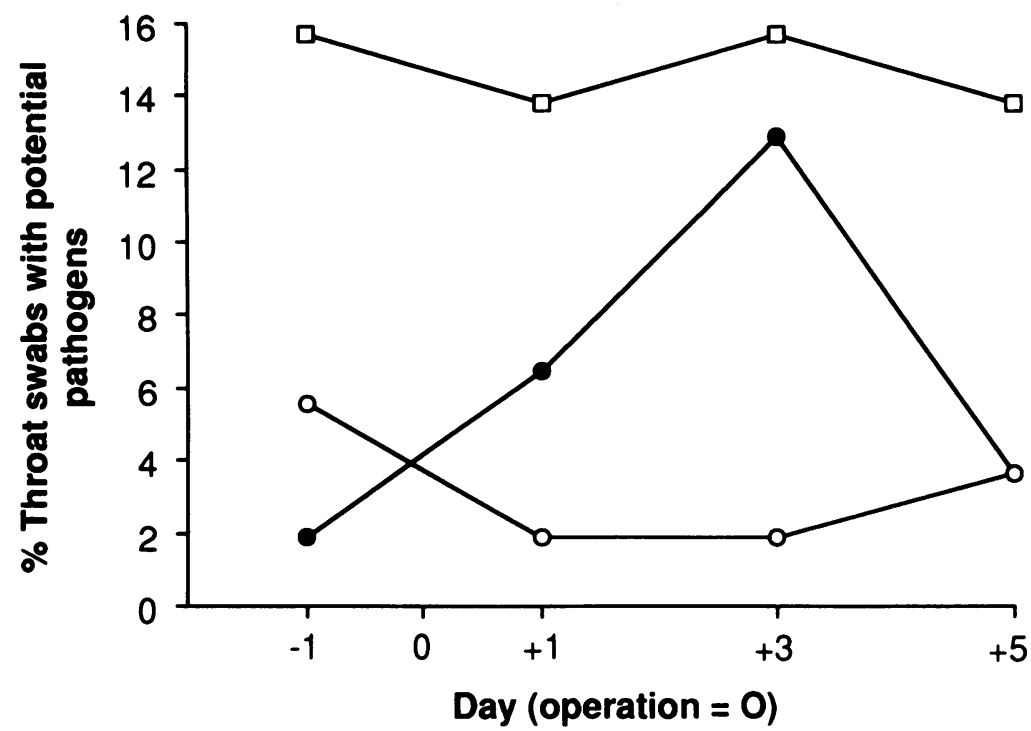

Isolation of potential pathogens from the oropharynx before and after operation. $\square \square$ Haemophilus influenzae; - - coliforms; - Streptococcus pneumoniae.

chitis was identified by production of sputum for three months of the year for two consecutive years.

Patients routinely received $1.5 \mathrm{~g}$ cefuroxime intravenously at induction of anaesthesia. Those patients undergoing gastric or colonic surgery also received $500 \mathrm{mg}$ metronidazole intravenously.

Approval was provided by Frenchay District Ethics Committee and verbal consent was obtained from all patients.

ANALYSIS

The incidence of preoperative $H$ influenzae and $S$ pneumoniae colonisation and of coliform colonisation on the third postoperative day was related to the development of chest infection and to cigarette smoking by the $\chi^{2}$ test with Yates's corrections.

\section{Results}

Of the 127 patients entering the study five patients declined to take part and 14 were excluded either because the full series of throat swabs was not obtained owing to early discharge (11 patients) or for other reasons (three patients). A full series of throat swabs from 108 patients was available for analysis.

There were 42 men and 66 women aged 1887 (mean 56) years. The operations were as follows: biliary (77), colonic (19), gastric (11), and splenectomy (1). The duration of surgery varied from 0.5 to 4.5 (mean 1.14 ) hours. Thirty seven patients (34\%) were smokers and 13 patients $(12 \%)$ had chronic bronchitis or chronic airflow obstruction.

Preoperatively 17 patients (16\%) were colonised by $H$ influenzae and the incidence was similar after operation. No patients had $H$ influenzae isolated from the oropharynx after operation who had not had it isolated before operation (figure). The isolation rate for $H$
Table 2 Preoperative Haemophilus influenzae colonisation in relation to postoperative chest infection

\begin{tabular}{lll}
\hline & \multicolumn{2}{l}{ Preoperative H influenzae } \\
\cline { 2 - 3 } & Present & Not present \\
\hline Chest infection & 9 & 15 \\
Total & $17^{\star}$ & $91^{\star}$ \\
\hline
\end{tabular}

${ }^{\star} \mathrm{p}<0.01$ ( $\chi^{2}$ test, Yates's correction).

influenzae was consistently higher with bacitracin chocolate agar than with chocolate agar and a bacitracin disc.

$S$ pneumoniae was present in six patients before operation, but only in two on the first and third postoperative days. In contrast, the incidence of coliform colonisation increased from two before operation to 14 on the third postoperative day, falling again to four patients on the fifth day. In addition, normal oropharyngeal bacterias were isolated in all patients before and after operation.

Postoperatively 24 patients ( $22 \%$ ) developed a chest infection, which became clinically apparent on the second, third, or fourth day. A sputum sample was obtained from 20 of these but in only eight patients was a definite pathogen isolated- $H$ influenzae from six patients and $S$ pneumoniae from two. Four of the patients with $H$ influenzae in the sputum at the time of infection had had this organism in the oropharynx; the two patients infected with $S$ pneumoniae had not had this organism isolated from the oropharynx. Coliform species were isolated from the sputum of a further nine patients, two of whom had had a coliform organism in the oropharynx. The mean length of postoperative stay was 8.3 (SD 5.4) days; it was increased in patients with chest infections to $10 \cdot 1$ days, compared with $7 \cdot 8$ days in those without infection, a non-significant difference $(p=0.071)$. Of the infections, 11 resolved spontaneously, 13 were treated with additional physiotherapy, and nine required additional medication, including antibiotics and bronchodilators.

Nine of 17 patients (53\%) with $H$ influenzae colonisation before operation developed infection compared with 15 of the $91(16.5 \%)$ noncolonised patients $(p<0.01)$ (table 2). There was also a significant relation between smoking and $H$ influenzae colonisation, 10 of 37 smokers $(27.0 \%)$ having preoperative $H$ influenzae colonisation compared with seven of 71 nonsmokers $(10 \%)(p<0.05)$. In the nonsmokers there was no significant relation between preoperative $H$ influenzae colonisation and the development of postoperative chest infection, though the number in this group is small. There was, however, a significant association between smoking and postoperative chest infection $(p<0.01)$.

No significant relation could be determined between the presence of $S$ pneumoniae and either infection or smoking, but the numbers are small. There was no association between the development of infection or smoking habit and the presence of coliform organisms on the third postoperative day. 


\section{Discussion}

Chest infection remains a common source of postoperative morbidity after elective upper abdominal surgery. The incidence has changed little over the past 50 years and ranges from $17 \%$ to $38 \%,{ }^{1-49}$ depending on the criteria used to diagnose infection. The predominant risk factors are cigarette smoking and smoking related lung disease. ${ }^{1-4}$

Growth of $H$ influenzae and of $S$ pneumoniae was investigated as these organisms have been identified as the major pathogens in previous studies, accounting for $72.9 \%$ to $78.3 \%{ }^{10}$ of postoperative chest infections. We also recorded the presence of coliform organisms because of the importance of Gram negative bacilli in the aetiology of nosocomial infection. ${ }^{112}$

Previous studies of postoperative chest infection have used a combination of fever, ${ }^{1213}$ cough productive of purulent sputum, ${ }^{12413}$ chest signs, ${ }^{13}$ and radiographic change ${ }^{2413}$ to diagnose chest infection. The production of purulent sputum indicates infection, though increased bronchial secretions in themselves may simply follow intubation and anaesthesia. Sputum purulence was therefore considered to be sufficient evidence of chest infection when only one other criteria was present. In the absence of purulent sputum four of the less specific criteria were required to make the diagnosis. With this system some patients with infection could possibly have been omitted but patients without infection are unlikely to have been included in the infection group.

The incidence of $H$ influenzae colonisation in the oropharynx preoperatively was $15 \cdot 7 \%$, which is similar to the $14.6 \%$ reported in a similar population of patients. Isolation was remarkably constant before and after surgery, allowing preoperative colonisation to be used as indicating a persistent state for the purposes of analysis.

The reason for the small fall in isolation rates for $S$ pneumoniae is not clear, but may represent a response to the antibiotic given. The numbers were too small for further analysis.

Coliform species were isolated in only two patients before operation. Both were unwell and a relation to severity of illness has been found previously. ${ }^{13}$ The incidence in the postoperative period increased, perhaps as a result of the cefuroxime administered as prophylaxis or the degree of illness (or both), though the incidence of $H$ influenzae colonisation was not altered significantly. In the few patients who received no antibiotics the isolation rates of $H$ influenzae were also unchanged.

The yield of microbiologically proved infection was low $(33 \%)$, as noted in previous studies of postoperative chest infection. ${ }^{2} \mathrm{We}$ were therefore unable to draw any conclusion about the relation between the preoperative presence of an oropharyngeal pathogen and the organism causing infection.

We have found a significant relation between $H$ influenzae carriage and both postoperative chest infection and cigarette smoking. Smoking increases the risk of postoperative chest infection and may also increase $\boldsymbol{H}$ influenzae colonisation independently. This is consistent with the finding in a previous study of oropharyngeal colonisation in the perioperative period, ${ }^{6}$ where an association was found between $H$ influenzae and chronic bronchitis. Alternatively, $H$ influenzae colonisation may be the primary risk factor for infection in smokers. Infection is more likely to be associated primarily with smoking, and this is supported by the low incidence of infection in patients with $H$ influen$z a e$ colonisation who do not smoke. No significant association could be detected between smoking or infection and isolation of pneumococci, but numbers were small. Nor was an association found between smoking or postoperative chest infection and the isolation of coliform species. Although coliform isolation increased after operation, we found no evidence that this was of clinical importance.

We have found a significant relation between preoperative $H$ influenzae colonisation and the development of postoperative chest infection in cigarette smokers. The importance of cigarette smoking as a risk factor for infection may be due at least in part to the presence of $H$ influenzae in the oropharynx. Whether efforts to eradicate $H$ influenzae would reduce the incidence of chest infection remains to be seen.

We thank the general surgeons and anaesthetists and the microbiology department of Frenchay Hospital for their cooperation in this study and Mr A O Hughes (Bristol University department of epidemiology) for assistance with the statistical analysis. JPD was in receipt of a grant from the Stanley Luff bequest.

1 Wightman JAK. A prospective survey of the incidence of postoperative pulmonary complications. Br J Surg 1968 55:85-91.

2 Garibaldi RA, Britt MR, Coleman ML, Reading JC, Pace NL. Risk factors for postoperative pneumonia. Am J Med 1981;70:677-80.

3 Dilworth JP, White RJ. Risk factors for postoperative chest infection after upper abdominal surgery [abstract]. Thorax 1989;44:856P.

4 Laszlo G, Archer GG, Darrell JH, Dawson JM, Fletcher $\mathrm{CM}$. The diagnosis and prophylaxis of pulmonary complications of surgical operations. Br J Surg 1973;60: plications

5 Nair P, Jani K, Sanderson PJ. Transfer of oropharyngeal bacteria into the trachea during endotracheal intubation. $J$ Hosp Infect 1986;8:96-103.

6 Tebbutt GM. Study of postoperative chest infections with particular emphasis on those caused by Haemophilus influenzea. J Clin Pathol 1986;39:78-83.

7 Dilworth JP, Pounsford JC, White RJ. The cough threshold after upper abdominal surgery. Thorax 1990;45:207-9.

8 Baber KG. A selective medium for the isolation of Haemophilus from the sputum. J Med Lab Technol 1969;26: 391-6.

9 King DS. Postoperative pulmonary complications. Surg Gynaecol Obstet 1933;56:43-50.

10 Wilkinson PJ, Ball AJ, Doran J, Gillespie WA, Orton VS. Routine laboratory assessment of postoperative chest infection: a prospective study. J Clin Pathol 1977;30: 417-20.

11 Bartlett JG, O'Keefe P, Tally FP, et al. The bacteriology of hospital acquired pneumonia. Arch Intern Med 1986;146: 868-71.

12 National Nosocomial Infections Study. Annual summary for 1979. Atlanta, Georgia: Centers for Disease Control, 1982:1-106.

13 Johanson WG, Pierce AK, Sanford JP. Changing pharyngeal bacterial flora of hospitalised patients. N Engl J Med 1969;281:1137-40. 